\title{
EDUCATION AND TRAINING A foundation interim year 1 sequential simulation experience and analysis of preparedness to practice early
}

\author{
Authors: Noah Havers, ${ }^{A}$ Alvaro Seebacher-Tomas ${ }^{A}$ and James Ashcroft ${ }^{B}$
}

This project involved the implementation of a simulation session followed by interviews to assess and improve foundation interim year 1 (FiY1) preparedness.

The session focused on the interpretation of investigations, clinical examinations, the implementation of management plans and appropriate escalation. Preparedness was measured quantitatively using Likert-type scales and qualitatively using interviews.

Following the simulation, there was a significant increase in median preparedness for giving treatment ( 3 vs $4 ; p=0.04$ ), paperwork ( 2 vs $4 ; p=0.03$ ) and independent, responsible working ( 3 vs $4.5 ; p=0.03$ ), before and after, respectively. Following the FiY1 period, participants demonstrated significant improvement in median preparedness for giving treatment ( 3 vs $4.5 ; p=0.01$ ), paperwork ( 2 vs $5 ; p=0.01$ ), independent, responsible working ( 3 vs $4.5 ; p=0.02$ ), and communication and teamworking ( 4 vs $5 ; p=0.01$ ), before and after, respectively.

This simulation and the FiY1 period increased preparedness. This study suggests that future medical apprenticeships should provide the same opportunities and responsibilities as the FiY1 programme.

KEYWORDS: curriculum, education environment, postgraduate, medicine, simulation

DOI: $10.7861 /$ fhj.2020-0183

\section{Introduction}

In 2020, the COVID-19 pandemic justified the early graduation of medical students in the UK enabling them to join the healthcare workforce as foundation interim year 1 (FiY1) doctors. ${ }^{1,2}$ This cohort of newly graduated junior doctors saw their curricula drastically changed, with many clinical placements being called off and medical school examinations postponed or cancelled. 3,4

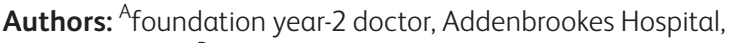
Cambridge, UK; B academic clinical fellow, University of Cambridge, Cambridge, UK
Prior to COVID-19, a survey found there was a significant discrepancy between medical student expectations and the reality of out-of-hours work. ${ }^{5} \mathrm{New}$ doctors often felt overwhelmed and apprehensive when dealing with acutely unwell patients. Lack of acute care exposure at medical school was identified as a substantial factor contributing towards this, despite management of acutely unwell patients being a requirement in the General Medical Council's (GMC) Outcomes for graduates. ${ }^{6,7}$

In 2020, many medical students missed the opportunity to experience a traditional apprenticeship period in which they would have shadowed graduate doctors and, therefore, this current cohort may feel less prepared than those in previous years. ${ }^{8}$ Safeguarding measures designed to support FiY1 doctors were put in place including direct supervision when assessing acutely unwell patients and work schedules restricted to daytime hours. ${ }^{9}$ FiY1 doctors were also not deployed in COVID-19 escalation wards and intensive care units, where possible. ${ }^{5}$

Sequential simulation allows the simulation of a series of key events or 'crunch points' that represent instances in the patient journey where physician intervention is essential. ${ }^{10}$ This study utilised a single session with multiple clinical scenarios to improve preparedness for early practice while assessing the preparedness of this new FiY1 cohort to practice early.

\section{Methods}

All newly graduated medical students were offered this simulation session. Participants were recruited through the local hospital junior doctors' forum and no power calculations were undertaken, although efforts were made to maximise the response rate by advertisement.

To undertake the session, participants were provided with a pager 'bleep', local guidelines, and relevant contact details of specialist departments or senior clinicians. Participants were asked to approach three major tasks as explained in Table 1. During this time, participants were also given several minor non-urgent tasks through the bleep system. We chose to include these small tasks to reflect the messy realities and challenges of clinical practice, rather than present a sanitised simulacrum. ${ }^{11}$ The students were also encouraged to contact 'senior support' as appropriate and after the simulation ended a debrief was undertaken using principles of 'diamond' feedback. ${ }^{12}$

A six-point Likert-type questionnaire ranging from 'very badly prepared' to 'very well prepared' was adapted from previous 
Table 1. Sequential simulation model

Introduction

Initial briefing

Major task structure

Minor task structure

Debrief

Participant evaluation
Participants informed about the simulation and give informed consent to participate. Pre-simulation questionnaire undertaken and demographic data collected.

Course structure explained and participant trained to use pager and relevant contact details of specialist departments or senior clinicians.

\section{Station 1}

New admission with pneumonia and pre-existing COPD, low suspicion of COVID-19.

Key investigations available for review include ABG (type 1 respiratory failure), CXR (pneumonia and COPD) and ECG (normal).

Escalation plan: bleep and discuss with the medical registrar for an urgent review.

\section{Station 2}

Ward-based deterioration with urinary infection causing agitation and confusion.

Key investigations available for review include blood test results (infection), CXR (normal) and urine dip (infection).

Escalation plan: bleep and discuss with the medical and microbiology specialist registrars for advice.

\section{Station 3}

Acute deterioration with suspected perforated abdominal viscera.

Patient played by actor requiring history taking and examination.

Key investigations available for review include VBG (metabolic acidosis), CXR (pneumoperitoneum) and ECG (normal).

Escalation plan: bleep and escalate to the surgical registrar.

Throughout major tasks, participants are were given several (one to five) minor non-urgent tasks through the bleep system. These tasks were designed to take less than 5 minutes and should be completable over the phone.

Participants undertook 'diamond' debrief conducted by three of the authors (all postgraduate doctors with 1-3 years' postgraduate experience).

Post-simulation questionnaire and semi-structured interview to explore the participants' experiences.

$\mathrm{ABG}=$ arterial blood gas; $\mathrm{COPD}=$ chronic obstructive pulmonary disease; $\mathrm{CXR}=$ chest $\mathrm{X}$-ray; $\mathrm{ECG}=$ electrocardiography; $\mathrm{VBG}=$ venous blood gases .

studies for preparedness in FY1s to investigate how prepared FiY1s felt approaching practice early pre-simulation, immediately post-simulation, and following 4 weeks of the FiY1 programme (supplementary material S1). ${ }^{13}$ The questionnaire assessed feelings of preparedness in eight areas integral to clinical practice. Semi-structured interviews were implemented following the simulation session and 4-week FiY1 programme to investigate participant learning experiences and preparedness for early practice. This interview was adapted from a previous study of preparedness in acute care (supplementary material S1). ${ }^{2}$

All interviewers were postgraduate doctors employed by Cambridge University Hospitals NHS Foundation Trust. Interviews were conducted with three people in the room: an interviewer, a scribe and the participant. A rapport was established with each participant by virtue of the simulation sessions as interviewers were present as facilitators during the session. Prior to the interview, the participant was fully informed of the goals of the interview, including the desire to produce a publication based on qualitative analysis of discussions and the use of anonymised direct quotes. ${ }^{14}$ Interviews were transcribed, quality assessed and anonymised before analysis.

Quantitative analysis of ordinal six-point Likert-type responses was undertaken by performing simple statistical analysis using SPSS version 23.0 (IBM, Armonk, USA). A Shapiro-Wilk analysis found the quantitative data to be non-parametric. Therefore, a Friedman's test over the three sessions was used with Wilcoxon signed-rank tests with Bonferroni correction for post hoc comparisons. Qualitative thematic analysis was undertaken using a six-phase approach of familiarisation: numbering of student responses, generating codes (independently done by two of the authors), searching for themes, reviewing themes, defining themes and report production. ${ }^{15}$

\section{Results}

A total of 12 FiY1s, all graduating in 2020, responded to the invitation to participate. This group comprised five men and seven women with a median age of 24 . Full background demographic information is available in supplementary material S2. All participants completed baseline, post-simulation and post-FiY1 (duration of 4 weeks) questionnaires and engaged in post-simulation and postFiY1 semi-structured interviews. At baseline, the preparedness of newly graduated FiY1s varied marginally between subscales with the majority of FiY1s feeling slightly prepared for all skills, except feeling slightly unprepared for giving treatment and independent, responsible working, and badly prepared for paperwork.

Following the sequential simulation session there was a significant $(p<0.05)$ improvement in preparedness for giving 


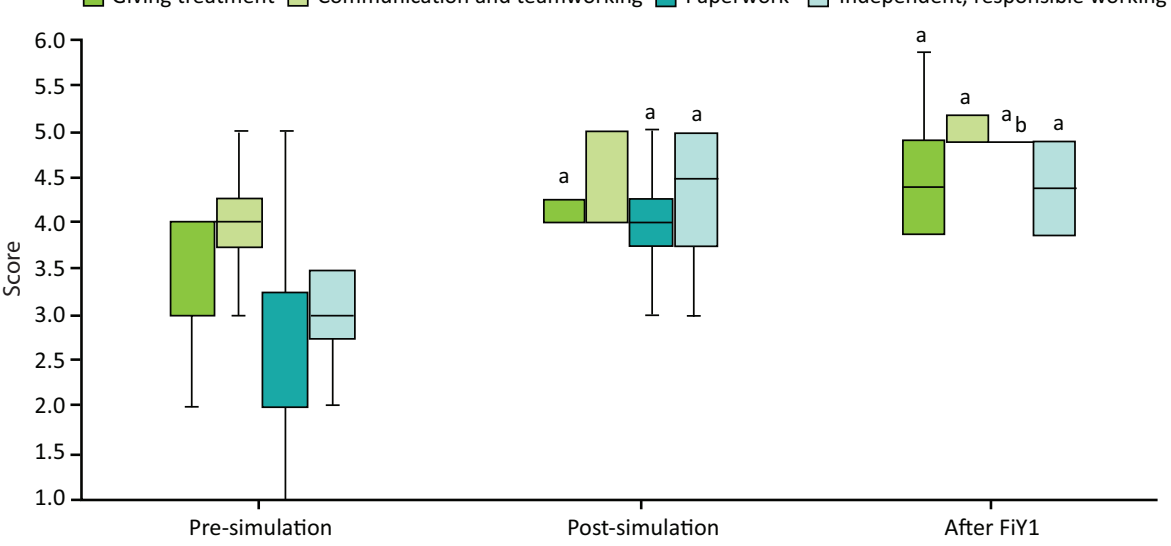

Fig 1. Foundation interim year-1 preparedness improvement. $a=$ $\mathrm{p}<0.05$ vs pre-simulation; $\mathrm{b}=\mathrm{p}<0.05$ vs post-simulation; $F$ FY 1 = foundation interim year-1 programme.

treatment, paperwork and independent, responsible working. Following 4 weeks of the FiY1 programme and when compared with baseline measures, FiY1s demonstrated significant improvement in preparedness for giving treatment; paperwork; independent, responsible working; and communication and teamworking (Fig 1; Table 2). The only preparedness subscale to demonstrate significant improvement between post-simulation and post-FiY1 measures was paperwork (Fig 1; Table 2).

During analysis of the semi-structured interview, major themes were identified both post-simulation (supplementary material S3) and following 4 weeks of FiY1 work (supplementary material S4). Pertinent or insightful points are illustrated by quotes. All participants gave positive feedback regarding the session. They described how the session accurately reflected real world learning:

In clinical school, we didn't get anything like this. It would have been really helpful ... Exams and lectures focused on very specific things whereas these scenarios were very open-ended.

They also discussed how it accurately replicated working under pressure:

Simulations involving real people are scary, your heart is genuinely pounding. It's a great mimic of doing things for the first time.

When interviewing participants following the 4-week FiY1 programme, it became apparent that most participants felt they lacked clarity on when, and how, to manage independently and escalate clinical scenarios appropriately, with one individual stating:

I have had a lot of learning on how to examine and historytaking. But I have had very little teaching of, once you have found the diagnosis, what do you do now?

Participants highlighted that they were able to rely on schema to assess cases:

The A to E structured approach really helped me feel more comfortable.

However, they were less confident in formulating management plans with independence:

Initially I would have not had a clue and I would have passed over to somebody else more senior, I would not have known where to go with the patient's management.
Some participants felt that simulations were still not adequate for real-life comparisons. One participant summarised this by stating:

Simulation will always bring extreme or classic cases, but there is a lack of 'in-between' cases where the diagnosis is not so clear cut.

Clinical placements and hands-on experience were consistently identified as a positive experience in equipping students for clinical practice during undergraduate training. One participant found that shadowing a doctor on their night shift was:

... an important experience and was transformative in terms of applying knowledge.

This is not something that is regularly found within medical school curricula.

Following FiY1, one substantial factor enhancing preparedness was the inclusion of FiY1 doctors within the central rota system. This allowed this new cohort of doctors to feel incorporated into their respective teams. One participant summarised this sentiment well when discussing their rota:

As they know I will be turning up tomorrow, they know it will be worth teaching me something now so that when I come tomorrow, I will be there to help them out with work. I was taught how to check tacrolimus levels, and this was something I was then able to do for the rest of the FiY1 period.

\section{Discussion}

This study suggests that sequential simulation prior to the introduction of clinical duties can enhance preparedness to practice for early medical graduates. Initially, FiY1 doctors felt underprepared to take on the role of a decision-maker when managing deteriorating patients. Through simulation and throughout the FiY1 programme, participants significantly improved their skills related to common responsibilities of FY1 doctors. Participants did not feel more prepared to implement skills such as history; examination, diagnosis and investigation; and patient safety, ethics and legal issues following simulation or FiY1. It is probable that these capabilities will develop as clinicians progress from junior to middle-grade physicians, where more responsibility is assumed for patient care. 
Table 2. Participant preparedness measures pre-simulation session, post-simulation session and after the 4-week foundation interim year-1 programme

\begin{tabular}{|c|c|c|c|c|}
\hline \multicolumn{2}{|l|}{ Preparedness outcome measures } & \multirow{2}{*}{$\begin{array}{l}\text { Median (IQR) } \\
3(3-4)\end{array}$} & \multirow[t]{2}{*}{$\begin{array}{l}\text { Significance vs } \\
\text { pre-simulation }\end{array}$} & \multirow[t]{2}{*}{$\begin{array}{l}\text { Significance vs } \\
\text { post-simulation }\end{array}$} \\
\hline Giving treatment & Pre-simulation & & & \\
\hline & Post-simulation & $4(4-4.75)$ & $0.04^{\mathrm{a}}$ & \\
\hline & Post-FiY1 & $4.5(4-5)$ & $0.01^{a}$ & 0.29 \\
\hline \multirow[t]{3}{*}{ Independent, responsible working } & Pre-simulation & $3(2.25-4.5)$ & & \\
\hline & Post-simulation & $4.5(3.25-5)$ & $0.03^{a}$ & \\
\hline & Post-FiY1 & $4.5(4-5)$ & $0.02^{a}$ & 0.54 \\
\hline \multirow[t]{3}{*}{ Dialoguing with patients } & Pre-simulation & $4(4-5)$ & & \\
\hline & Post-simulation & $5(4-5)$ & 0.31 & \\
\hline & Post-FiY1 & $5(5-5)$ & 0.07 & 0.40 \\
\hline \multirow[t]{3}{*}{ History, examination, diagnosis and investigation } & Pre-simulation & $4(4-4.75)$ & & \\
\hline & Post-simulation & $4(4-5)$ & 0.54 & \\
\hline & Post-FiY1 & $4(4-5)$ & 1.00 & 1.00 \\
\hline \multirow[t]{3}{*}{ Communication and team working } & Pre-simulation & $4(3.25-4.75)$ & & \\
\hline & Post-simulation & $5(4-5)$ & 0.07 & \\
\hline & Post-FiY1 & $5(5-5.75)$ & $0.01^{a}$ & 0.06 \\
\hline \multirow[t]{3}{*}{ Procedural skills } & Pre-simulation & $4(3-4.75)$ & & \\
\hline & Post-simulation & $4(4-4.75)$ & 0.95 & \\
\hline & Post-FiY1 & $4(5.575)$ & 0.79 & 1.00 \\
\hline \multirow[t]{3}{*}{ Patient safety, ethics and legal issues } & Pre-simulation & $4(3-4.75)$ & & \\
\hline & Post-simulation & $4(3.25-5)$ & 0.77 & \\
\hline & Post-FiY1 & $4(3.25-5)$ & 0.62 & 1.00 \\
\hline \multirow[t]{3}{*}{ Paperwork } & Pre-simulation & $2(2-3.75)$ & & \\
\hline & Post-simulation & $4(3.25-4.75)$ & $0.03^{a}$ & \\
\hline & Post-FiY1 & $5(5-5)$ & $0.01^{a}$ & $0.02^{a}$ \\
\hline
\end{tabular}

This cohort is a previously unstudied group and represents physicians graduating earlier than preceding cohorts to assist in the pandemic response. Whereas previously students underwent an apprenticeship, wherein they were assigned to wards and learnt by shadowing doctors, the FiY1 period placed senior students as an essential component of an acute care team with tasks to carry out under varying supervision to ensure the safe care of patients on the ward. Previous studies have suggested that the apprenticeship shadowing period for medical students is too passive. Therefore, the implementation of an FiY1 placement may have given early graduates a more representative and active learning experience in the workplace. ${ }^{2}$ This was reflected in the way our participants felt in their second interview, having worked for 4 weeks as FiY1s. The FiY1 programme allowed for a gradual entry to practice due to restricted responsibilities and oversight by trainees throughout most of the period, while also giving a fixed schedule and clear duties for the participants, which most considered was a perfect introduction to practice. We believe a similar FiY1 programme, built on integrated responsibility and appropriate incentives, which would allow for a structured introduction into the junior doctor workforce, should be retained in the future, regardless of pandemic status, as an opportunity to improve medical training in the long term.

This study focused on preparedness to practice with one important limitation being the lack of a pragmatic objective measure of performance. It cannot be assumed that self-reported confidence is reflective of competence to practice. ${ }^{16}$ Furthermore, it is difficult to ascertain whether simulated preparedness translates to improved clinical practice. It is particularly challenging to assess whether changes in preparedness were truly related to simulation or FiY1 experiences, considering the abundance of training experiences available to the participants over this period. Therefore, future longitudinal studies focused on this cohort and future adaptations of medical curricula must be undertaken with robust clinical outcomes. Due to the limited introduction of the FiY1 role, both temporal and longitudinal aspects of this study were limited and only a small number of early graduates participated. However, the important conclusions drawn from this cohort should still influence the design of future undergraduate apprenticeship periods. 


\section{Conclusion}

Participants of this sequential simulation session and 4-week FiY1 programme gained confidence and reported significant improvements in their preparedness to practice as junior clinicians. Following undergraduate training, medical students felt unprepared to carry out independent work and become key decision makers when approaching deteriorating patients. The incorporation of senior medical students into a role of responsibility as FiY1s, replacing a passive shadowing placement, allowed new doctors to prepare to practice in a safe and supportive environment. This study suggests that future senior medical student apprenticeship placements should be replaced by an FiY1-type rotation.

\section{Supplementary material}

Additional supplementary material may be found in the online version of this article at www.rcpjournals.org/clinmedicine: S1 - Likert-type scale for rating of preparedness pre-simulation, post-simulation and post-FiY1 programme, and interview topic guide post-simulation and post-FiY1 programme.

S2 - Participant demographics.

S3 - Experience of preparedness post-simulation session.

S4 - Experience of preparedness following a 4-week FiY1 programme.

\section{Acknowledgements}

The authors wish to thank the facilitators Stephanie Goh, Victoria Hudson and Elena Plesca involved in the sequential simulation sessions, Jack Pottle and Oxford Medical Simulation for providing our participants with virtual reality simulation scenarios and the students for their participation and feedback. When conceptualising this study, James Ashcroft tweeted and received guidance (https:// twitter.com/JamesAshcroftMD/status/1255954377405337600) from Anthony Codd @DoctorCodd, Simon Fleming @OrthopodReg, Jonny Guckian@JonnyGucks, David Hettle @dave_hettle, Viktoria Joynes @ViktoriaCT], Kay Leedham-Green @doctorkayleigh, Catherine Lovegrove@CELovegrove, Nabilah Mayat @NabilahMayat, Alice McCloskey @DrAliceMcC, Harry Spiers @harryvmspiers, Tanya Ta@veggieequallife, and Jordan Tsigarides@VRMedicsNorwich. Aimee Charnell @aimeecharnell gave her time and further invaluable advice by twitter and phone which the authors are very grateful for.

\section{References}

1 Department of Health and Social Care. Health and Social Care secretary responds to NHS workforce statistics. GOV.UK, 2020. www.gov.uk/government/news/health-and-social-care-secretaryresponds-to-nhs-workforce-statistics [Accessed 26 August 2020].
2 Iacobucci G. COVID-19: medical schools are urged to fast-track final year students. BMJ 2020;368:m1064.

3 O'Byrne L, Gavin B, McNicholas F. Medical students and COVID-19: the need for pandemic preparedness. J Med Ethics 2020;46:623-6.

4 Arora A, Solomou G, Bandyopadhyay S et al. Adjusting to Disrupted Assessments, Placements and Teaching (ADAPT): a snapshot of the early response by UK medical schools to COVID19. medRxiv 2020.07.29.20163907.

5 Brown M, Shaw D, Sharples S et al. A survey-based cross-sectional study of doctors' expectations and experiences of non-technical skills for Out of Hours work. BMJ Open 2015;5:e006102.

6 Burridge S, Shanmugalingam T, Nawrozzadeh F et al. A qualitative analysis of junior doctors' journeys to preparedness in acute care. BMC Med Educ 2020;20:12.

7 General Medical Council. Outcomes for graduates. GMC, 2020. www.gmc-uk.org/education/standards-guidance-and-curricula/ standards-and-outcomes/outcomes-for-graduates/outcomes-forgraduates [Accessed 26 August 2020].

8 Van Hamel C, Jenner LE. Prepared for practice? A national survey of UK foundation doctors and their supervisors. Med Teach 2014;37:181-8.

9 Ashcroft J, Byrne MHV, Brennan PA, Davies RJ. Preparing medical students for a pandemic: a systematic review of student disaster training programmes. Postgrad Med J 2020 [Epub ahead of print].

10 Powell P, Sorefan Z, Hamilton S, Kneebone R, Bello F. Exploring the potential of sequential simulation. Clin Teach 2015;13:112-8.

11 Kneebone R. PhD perspective: Simulation and transformational change: the paradox of expertise. Acad Med 2009;84:954-7.

12 Jaye P, Thomas L, Reedy G. "The Diamond": A structure for simulation debrief. Clin Teach 2015;12:171-5.

13 Miles S, Kellett J, Leinster SJ. Medical graduates' preparedness to practice: a comparison of undergraduate medical school training. BMC Med Educ 2017; 17:33.

14 Tong A, Sainsbury P, Craig J. Consolidated criteria for reporting qualitative research (COREQ): a 32-item checklist for interviews and focus groups. Int J Qual Health Care 2007;19:349-57.

15 Braun V, Clarke V. Using thematic analysis in psychology. Qualitative Research in Psychology 2006;3:77-101.

16 Brinkman D, Tichelaar ], Agtmael MA, De Vries TPGM, Richir MC. Self-reported confidence in prescribing skills correlates poorly with assessed competence in fourth-year medical students. J Clin Pharmacol 2015:55:825-30.

Address for correspondence: Dr James Ashcroft, University of Cambridge Department of Surgery, Addenbrookes Hospital, Hills Road, Cambridge CB2 0QQ, UK.

Email: jaa72@cam.ac.uk

Twitter: @JamesAshcroftMD 